ESAIM: PROCEEDINGS, December 2013, Vol. 42, p. 20-33

A. Heibig, F. Filbet, L. I. Palade, Editors

\title{
THE SINGULAR DYNAMIC METHOD FOR DYNAMIC CONTACT OF THIN ELASTIC STRUCTURES
}

\author{
Cédric Pozzolini ${ }^{1}$, Yves Renard ${ }^{2}$ et Michel Salaün ${ }^{3}$
}

\begin{abstract}
This paper adresses the approximation of the dynamic impact of thin elastic structures. The principle of the presented method is the use of a singular mass matrix obtained by different discretizations of the deflection and velocity. The obtained semidiscretized problem is proved to be well-posed and energy conserving. The method is applied on some membrane, beam and plate models and associated numerical experiments are discussed.
\end{abstract}

\section{INTRODUCTION}

When the discretization of impact of elastic structures is addressed, it is generally noted that the vast majority of traditional time integration schemes show spurious oscillations on the contact displacement and stress (see for instance $[7,8,11]$ ). Moreover, these oscillations do not disappear when the time step decreases. Conversely, they tend to increase which is a characteristic of order two hyperbolic equations with unilateral constraints that makes it very difficult to build stable numerical schemes. These difficulties have already led to many researches under which a variety of solutions were proposed. Some of them consist in adding damping terms (see [27] for instance), but with a loss of accuracy on the solution, or to implicit the contact stress $[4,5]$ but with a loss of kinetic energy which could be independent of the discretization parameters (see the numerical experiments). Some energy conserving schemes have also been proposed in $[7,8,10,15,16,28]$. Unfortunately, these schemes, although more satisfactory than most of the other ones, lead to large oscillations on the contact stress. Besides, most of them do not strictly respect the constraint.

In this paper, we describe a class of methods whose principle is to make different approximations of the solution and of its time derivative. Such a principle was already studied for linear elastodynamics in [9]. Compared to the classical space semi-discretization, this corresponds to a singular modification of the mass matrix. In this sense, it is in the same class of methods than the mass redistribution method proposed in $[11,12]$ for elastodynamic contact problems. The main feature is to provide a well-posed space semi-discretization. The numerical tests show that it has a crucial influence on the stability of standard schemes and on the quality of the approximation, especially for the computation of Lagrange multipliers corresponding to the constraints.

The method is first described on an abstract hyperbolic equation on which the well-posedness of the semi-discretized problem by finite elements is proven. The method is then described on several models of thin elastic structures, namely membrane, Euler-Bernouilli beam and Kirchhoff-Love plate models. Some numerical tests for all these models are given and discussed. Finally, we present some perspectives and open problems.

\footnotetext{
1 Université de Lyon, CNRS, INSA-Lyon, ICJ UMR5208, F-69621 Villeurbanne, France, cedric.pozzolini@insalyon.fr

2 Université de Lyon, CNRS, INSA-Lyon, ICJ UMR5208, LaMCoS UMR5259, F-69621 Villeurbanne, France, yves.renard@insa-lyon.fr

${ }^{3}$ Université de Toulouse; INSA, UPS, EMAC, ISAE; ICA (Institut Clément Ader), 10 Avenue Edouard Belin, F-31055 Toulouse, France, michel.salaun@isae.fr
} 


\section{The Method For AN ABSTRACT HyPERBolic EQUATION}

The method is introduced in [25] on the following abstract hyperbolic problem. Let $\Omega \subset \mathbb{R}^{d}$ be a Lipschitz domain and $H=L^{2}(\Omega)$ the standard Hilbert space of square integrable functions on $\Omega$. Let $W$ be a Hilbert space such that $W \subset H \subset W^{\prime}$, with dense compact and continuous inclusions and let $A: W \rightarrow W^{\prime}$ be a linear self-adjoint elliptic continuous operator. We consider the following problem

$$
\left\{\begin{array}{l}
\text { Find } u:[0, T] \rightarrow K \text { such that } \\
\frac{\partial^{2} u}{\partial t^{2}}(t)+A u(t) \in f-N_{K}(u(t)), \text { for a.e. } t \in(0, T], \\
u(0)=u_{0}, \frac{\partial u}{\partial t}(0)=v_{0},
\end{array}\right.
$$

where $K$ is a closed convex nonempty subset of $W, f \in W^{\prime}, u_{0} \in K, v_{0} \in H, T>0$ and $N_{K}(u)$ is the normal cone to $K$ defined by (see for instance [3] for more details)

$$
N_{K}(u)=\left\{\begin{array}{l}
\emptyset, \text { if } u \notin K, \\
\left\{f \in W^{\prime}:\langle f, w-u\rangle_{W^{\prime}, W} \leq 0, \forall w \in K\right\} \quad, \quad \text { if } u \in K .
\end{array}\right.
$$

This means that $u(t)$ satisfies the second order hyperbolic equation and is constrained to remain in the convex $K$. There is no general result of existence nor uniqueness for the solution to this problem. Some existence results for a scalar Signorini problem can be found in [14,17]. Introducing now the linear and bilinear symmetric maps

$$
l(v)=\langle f, v\rangle_{W^{\prime}, W}, a(u, v)=\langle A u, v\rangle_{W^{\prime}, W}
$$

Problem (1) can be rewritten as the following variational inequality:

$$
\left\{\begin{array}{l}
\text { Find } u:[0, T] \rightarrow K \text { such that for a.e. } t \in(0, T] \\
\left\langle\frac{\partial^{2} u}{\partial t^{2}}(t), w-u(t)\right\rangle=l(u(t), w-u(t)) \geq l(w-u(t)), \forall w \in K, \\
u(0)=u_{0}, \frac{\partial u}{\partial t}(0)=v_{0} .
\end{array}\right.
$$

Note that the terminology "variational inequality" is used here in the sense that Problem (1) derives from the conservation of the energy functional

$$
J(t)=\frac{1}{2} \int_{\Omega}\left(\frac{\partial u}{\partial t}(t)\right)^{2} d x+\frac{1}{2} a(u(t), u(t))-l(u(t))+I_{K}(u(t))
$$

where $I_{K}(u(t))$ is the convex indicator function of $K$. However, it is generally not possible to prove that each solution to Problem (2) is energy conserving, due to the weak regularity involved.

\section{Approximation AND Well-Posedness Result}

The aim of this section is to present well-posed space semi-discretizations of Problem (2). The adopted strategy is to use a Galerkin method with different approximations of $u$ and of $v=\frac{\partial u}{\partial t}$. Let $W^{h}$ and $H^{h}$ be two finite dimensional vector subspaces of $W$ and $H$ respectively. Let $K^{h} \subset W^{h}$ be a closed convex nonempty approximation of $K$. The proposed approximation of Problem (2) is the following mixed approximation:

$$
\left\{\begin{array}{l}
\text { Find } u^{h}:[0, T] \rightarrow K^{h} \text { and } v^{h}:[0, T] \rightarrow H^{h} \text { such that } \\
\int_{\Omega} \frac{\partial v^{h}}{\partial t}\left(w^{h}-u^{h}\right) d x+a\left(u^{h}, w^{h}-u^{h}\right) \geq l\left(w^{h}-u^{h}\right), \forall w^{h} \in K^{h}, \forall t \in(0, T], \\
\int_{\Omega}\left(v^{h}-\frac{\partial u^{h}}{\partial t}\right) q^{h} d x=0, \forall q^{h} \in H^{h}, \forall t \in(0, T], \\
u^{h}(0)=u_{0}^{h}, \quad v^{h}(0)=v_{0}^{h},
\end{array}\right.
$$


where $u_{0}^{h} \in K^{h}$ and $v_{0}^{h} \in H^{h}$ are some approximations of $u_{0}$ and $v_{0}$ respectively. Of course, when $H^{h}=W^{h}$, this corresponds to a standard Galerkin approximation of Problem (2).

Let $\varphi_{i}, 1 \leq i \leq N_{W}$, and $\psi_{i}, 1 \leq i \leq N_{H}$, be some basis of $W^{h}$ and $H^{h}$ respectively, and let the matrices $\bar{A}, B$ and $C$, of sizes $N_{W} \times N_{W}, N_{H} \times N_{W}$ and $N_{H} \times N_{H}$ respectively, and the vectors $L, U$ and $V$, of size $N_{W}, N_{W}$ and $N_{H}$ respectively, be defined by

$$
\begin{gathered}
A_{i, j}=a\left(\varphi_{i}, \varphi_{j}\right), \quad B_{i, j}=\int_{\Omega} \psi_{i} \varphi_{j} d x \quad, \quad C_{i, j}=\int_{\Omega} \psi_{i} \psi_{j} d x, \\
L_{i}=l\left(\varphi_{i}\right), u^{h}=\sum_{i=1}^{N_{W}} U_{i} \varphi_{i} \quad, \quad v^{h}=\sum_{i=1}^{N_{H}} V_{i} \psi_{i} .
\end{gathered}
$$

Then, $U$ and $V$ are linked by the equation $C V(t)=B \dot{U}(t)$. So $V$ can be eliminated since $C$ is always invertible, which leads to the relation $V(t)=C^{-1} B \dot{U}(t)$. Consequently, Problem (3) can be rewritten as

$$
\left\{\begin{array}{l}
\text { Find } U:[0, T] \rightarrow \bar{K}^{h} \text { such that } \\
(W-U(t))^{T}(M \ddot{U}(t)+A U(t)) \geq(W-U(t))^{T} L, \forall W \in \bar{K}^{h}, \forall t \in(0, T] \\
U(0)=U_{0}, B \dot{U}(0)=C V_{0}
\end{array}\right.
$$

In comparison with the standard approximation where $H^{h}=W^{h}$, the only difference introduced by the presented method is to replace the standard mass matrix $\left(\int_{\Omega} \varphi_{i} \varphi_{j} d x\right)_{i, j}$ by $M=B^{T} C^{-1} B$. In the interesting cases where $\operatorname{dim}\left(H^{h}\right)<\operatorname{dim}\left(W^{h}\right)$, it corresponds to replace the standard invertible mass matrix by a singular one.

Although the analysis could probably be extended to more complex situations, we assume that $K^{h}$ is defined by a finite number of linear constraints as

$$
K^{h}=\left\{w^{h} \in W^{h}: g^{i}\left(w^{h}\right) \leq \alpha^{i}, 1 \leq i \leq N_{g}\right\}
$$

where $\alpha^{i} \in \mathbb{R}$ and $g^{i}: W^{h} \rightarrow \mathbb{R}, 1 \leq i \leq N_{g}$, are some linearly independent linear maps. Of course, this restricts the possibilities concerning the convex $K$ since $K^{h}$ is supposed to be an approximation of $K$. With vector notations, this leads to

$$
\bar{K}^{h}=\left\{W \in \mathbb{R}^{N_{W}}:\left(G^{i}\right)^{T} W \leq \alpha_{i}, 1 \leq i \leq N_{g}\right\}
$$

where $G^{i} \in \mathbb{R}^{N_{W}}$ are such that $g^{i}\left(w^{h}\right)=\left(G^{i}\right)^{T} W, 1 \leq i \leq N_{g}$. We will also denote by $G$ the $N_{W} \times N_{g}$ matrix whose components are

$$
G_{i j}=\left(G^{i}\right)_{j}
$$

Let us consider the subspace $F^{h}$ of $W^{h}$ defined by

$$
F^{h}=\left\{w^{h} \in W^{h}: \int_{\Omega} w^{h} q^{h}=0, \forall q^{h} \in H^{h}\right\} .
$$

Then, the corresponding set $F=\left\{W \in \mathbb{R}^{N_{W}}: \sum_{i=1}^{N_{W}} W_{i} \varphi_{i} \in F^{h}\right\}$ is such that $F=\operatorname{Ker}(B)$. In this framework, we consider the following condition:

$$
\inf _{\substack{Q \in \mathbb{R}^{N} \\ Q \neq 0}} \sup _{\substack{W \in F \\ W \neq 0}} \frac{Q^{T} G W}{\|Q\|\|W\|}>0
$$

where $\|Q\|$ and $\|W\|$ stand for the Euclidean norm of $Q$ in $\mathbb{R}^{N_{g}}$ and $W$ in $\mathbb{R}^{N_{W}}$ respectively. This condition is equivalent to the fact that the linear maps $g^{i}$ are independent on $F^{h}$ and also to the fact 
that $G$ is surjective on $F$. A direct consequence is that it implies $\operatorname{dim}\left(F^{h}\right) \geq N_{g}$ and consequently

$$
\operatorname{dim}\left(H^{h}\right) \leq \operatorname{dim}\left(W^{h}\right)-N_{g}
$$

This again prescribes some conditions on the approximations which link $W^{h}, H^{h}$ and also $K^{h}$. We will see in Section 3 that this condition can be satisfied for interesting practical situations. We can now prove the following result:

Theorem 1. If $W^{h}, H^{h}$ and $K^{h}$ satisfy condition (5), then Problem (4) admits a unique solution. Moreover, this solution is Lipschitz-continuous with respect to $t$.

Proof of this theorem can be found in [25]. In particular, it is based on the following result allowing a decomposition of the solution:

Lemma 1. If $W^{h}, H^{h}$ and $K^{h}$ satisfy condition (5), then there exists a sub-space of $\mathbb{R}^{N_{W}}$, say $F^{c}$, such that $F^{c} \subset \operatorname{Ker}(G)$ and such that $F$ and $F^{c}$ are complementary sub-spaces.

Moreover, the following energy conservation is proved:

Theorem 2. If $W^{h}, H^{h}$ and $K^{h}$ satisfy condition (5), then the solution $U(t)$ to Problem (4) is energy conserving in the sense that the discrete energy

$$
J^{h}(t)=\frac{1}{2} \dot{U}^{T}(t) M \dot{U}(t)+\frac{1}{2} U^{T}(t) A U(t)-U^{T}(t) L
$$

is constant with respect to $t$.

\section{Applichtion to a membrane model}

This section provides a simple but interesting situation for which some consistent approximations satisfy the condition (5). When $W=H^{1}(\Omega)$ and $K=\{w \in W: w \geq 0$ a.e. on $\Omega\}$, we consider the following problem

$$
\left\{\begin{array}{l}
\text { Find } u:[0, T] \rightarrow K \text { such that } \\
\frac{\partial^{2} u}{\partial t^{2}}(t)-\Delta u(t) \in f-N_{K}(u(t)) \text { in } \Omega, \text { for a.e. } t \in(0, T] \\
\frac{\partial u}{\partial n}=0 \text { on } \Gamma_{N}, \\
u=0 \text { on } \Gamma_{D}, \\
u(0)=u_{0}, \frac{\partial u}{\partial t}(0)=v_{0},
\end{array}\right.
$$

where $\Gamma_{N}$ and $\Gamma_{D}$ is a partition of $\partial \Omega, \Gamma_{D}$ being of non zero measure in $\partial \Omega$. This models for instance the contact between an antiplane elastic structure with a rigid foundation or a stretched drum membrane under an obstacle condition. In this situation, the mass redistribution method presented in [12] is not usable since the area subjected to potential contact is the whole domain. Consequently, this method would lead to suppress the mass on the whole domain which is a non consistent drastic change of the problem.

We build now the approximation spaces thanks to finite element method. Let $\mathscr{T}^{h}$ a regular triangular mesh of $\Omega$ (in the sense of Ciarlet [2], $h$ being the diameter of the largest element) and $W^{h}$ be the following $P_{1}+$ finite element space

$$
W^{h}=\left\{w^{h} \in \mathscr{C}^{0}(\Omega): w^{h}=\sum_{a_{i} \in \mathscr{A}} w_{i} \varphi_{i}+\sum_{T \in \mathscr{T}^{h}} w_{T} \varphi_{T}\right\},
$$

where $\mathscr{A}$ is the set of the vertices of the mesh which do not lie on $\Gamma_{D}$. Then, $\varphi_{i}, a_{i} \in \mathscr{A}$, are the piecewise linear functions satisfying $\varphi_{i}\left(a_{j}\right)=\delta_{i j}$, where $\delta_{i j}$ is Kronecker symbol, i.e. the shape 
functions of a $P_{1}$ Lagrange finite element method on $\mathscr{T}^{h}$. Each function $\varphi_{T}, T \in \mathscr{T}^{h}$, is the cubic bubble function whose support is $T$. Let $H^{h}$ be the $P_{0}$ finite element space

$$
H^{h}=\left\{v^{h} \in L^{2}(\Omega): v^{h}=\sum_{T \in \mathscr{T}^{h}} v_{T} \mathbb{I}_{T}\right\},
$$

and, finally, let $K^{h}$ be defined as

$$
K^{h}=\left\{w^{h} \in W^{h}: w^{h}\left(a_{i}\right) \geq 0, \text { for all } a_{i} \in \mathscr{A}\right\},
$$

which means that the constraints are only prescribed at the vertices of the mesh. Then, it is proved in [25] that this choice of $W^{h}, H^{h}$ and $K^{h}$ satisfies condition (5).

\section{EXtension tO THE VIBRO-IMPACT OF STRUCTURES ON RIGID OBSTACLES}

\subsection{Case of a beam}

In [23], the method is applied to the fourth order problem of the dynamical evolution of an Euler-Bernouilli beam evolving between two rigid obstacles. The considered unknown is the vertical deflection, which is constrained to belong to

$$
K=\left\{w \in H^{2}(0, L): g_{1}(x) \leq w(x) \leq g_{2}(x), \text { for all } x \in[0, L]\right\},
$$

where $g_{1}$ and $g_{2}$ are two maps from $[0, L]$ to $\overline{\mathbb{R}}:=\mathbb{R} \cup\{-\infty,+\infty\}$ such that

$$
g_{1}(x)<0<g_{2}(x), \forall x \in[0, L]
$$

These maps denote the position of the obstacles. If $u(x, t)$ is the vertical deflection, the strong formulation of the problem, in the case of a clamped-free beam, reads as

$$
\left\{\begin{array}{l}
\text { Find } u:[0, T] \rightarrow K \text { such that } \\
\rho S \frac{\partial^{2} u}{\partial t^{2}}(t)+E I \frac{\partial^{4} u}{\partial x^{4}}(x, t) \in f-N_{K}(u(t)), \quad \forall(x, t) \in[0, L] \times(0, T], \\
u(x, 0)=u_{0}(x), \quad \frac{\partial u}{\partial t}(x, 0)=v_{0}(x), \forall x \in[0, L], \\
u(0, t)=\frac{\partial u}{\partial x}(0, t)=\frac{\partial^{2} u}{\partial x^{2}}(L, t)=\frac{\partial^{3} u}{\partial x^{3}}(L, t)=0 \quad, \quad \forall t \in(0, T],
\end{array}\right.
$$

where $\rho>0$ is the mass density, $E$ is the Young modulus, while $S$ and $I$ are the surface and the inertial momentum of the beam section, respectively.

The weak form of this problem can be written as

$$
\left\{\begin{array}{l}
\text { Find } u:[0, T] \rightarrow K_{0} \text { and } v:[0, T] \rightarrow L^{2}(0, L) \text { such that for a.e. } t \in(0, T] \\
\int_{0}^{L}\left[\rho S \frac{\partial v}{\partial t}(w-u)+E I \frac{\partial^{2} u}{\partial x^{2}} \frac{\partial^{2}(w-u)}{\partial x^{2}}\right] d x \geq \int_{0}^{L} f(w-u) d x \quad, \forall w \in K_{0} \\
\int_{0}^{L}\left(v-\frac{\partial u}{\partial t}\right) q d x=0 \quad, \forall q \in L^{2}(0, L), \\
u(x, 0)=u_{0}(x) \in K_{0} \quad, \quad v(x, 0)=v_{0}(x) \in L^{2}(0, L) \quad, \quad \forall x \in[0, L]
\end{array}\right.
$$

where $K_{0}=\left\{w \in K: w(0)=w^{\prime}(0)=0\right\}$.

To build the finite element method, it is introduced a partition of $[0, L]$ into $N$ subintervals of length $h=L / N$, built on nodes $x_{i}=i h$, for $0 \leq i \leq N$. As node $x_{0}=0$ is clamped, we will omit it from now on and consider that index $i$ varies between 1 and $N$. Otherwise, it would introduce small modifications in the following. So, at each node $x_{i}$ are associated two Hermite piecewise cubic functions, say $\phi_{2 i-1}$ and $\phi_{2 i}$, defined for $1 \leq i \leq N$ by

$$
\phi_{2 i-1}\left(x_{j}\right)=\delta_{i j} \quad \text { and } \quad \phi_{2 i-1}^{\prime}\left(x_{j}\right)=0 \quad, \quad \phi_{2 i}\left(x_{j}\right)=0 \quad \text { and } \quad \phi_{2 i}^{\prime}\left(x_{j}\right)=\delta_{i j} .
$$


Moreover, functions $\phi_{j}$ are chosen of class $\mathcal{C}^{1}$ on $[0, L]$, which insures that each $\phi_{j}$ belongs to the continuous space $W=\left\{H^{2}(0, L): w(0)=w^{\prime}(0)=0\right\}$. Hence, displacement $w^{h}$ reads

$$
w^{h}(x)=\sum_{i=1}^{N} w_{2 i-1}^{h} \phi_{2 i-1}(x)+\sum_{i=1}^{N} w_{2 i}^{h} \phi_{2 i}(x)
$$

and coefficient $w_{2 i-1}^{h}$ gives the value of $w^{h}$ at node $x_{i}$ while $w_{2 i}^{h}$ gives the value of its derivative at the same node. The approximation space for displacements is then

$$
W^{h}=\operatorname{span}\left\{\phi_{j}, 1 \leq j \leq 2 N\right\}
$$

Let us now explain how the approximation $K^{h}$ of $K_{0}$ is obtained. Following the idea of the previous section, unilateral constraints are only considered at the nodes of the mesh. It means convex $K^{h}$ is

$$
K^{h}=\left\{w^{h} \in W^{h} / g_{1}\left(x_{i}\right) \leq w^{h}\left(x_{i}\right) \leq g_{2}\left(x_{i}\right), \forall i \in[0, N]\right\}
$$

With vector notations, setting $\alpha_{i}^{-} \equiv g_{1}\left(x_{i}\right)$ and $\alpha_{i}^{+} \equiv g_{2}\left(x_{i}\right)$ for all $i$, this space may be written (we keep the same notation for simplicity)

$$
K^{h}=\left\{W \in \mathbb{R}^{N_{W}} / \alpha_{i}^{-} \leq\left(G^{i}\right)^{T} W \leq \alpha_{i}^{+}, \forall i \in[0, N]\right\}
$$

where $G^{i}$ is the vector of $\mathbb{R}^{N_{W}}$ such that $\left(G^{i}\right)^{T} W=w^{h}\left(x_{i}\right)$, for all node $x_{i}$.

It is proved in [23] that such a nodal contact condition together with the use of the cubic Hermite element for the deflection and either a piecewise constant finite element method, or a continuous linear one, for the velocity satisfy the inf-sup condition (5).

Remark 1. Since we deal with a fourth order problem with respect to the space derivative, it is not possible to consider a linear space approximation. In fact, for this beam model, we use the classical Hermite third degree polynomials to approximate the numerical displacement. In the above approximation of $K$, as we consider only constraints on node displacements, the effect of the derivatives, namely the curvature, is not taken into account. Then, in this framework, the beam could cross the obstacle between two nodes, but we shall neglect this aspect in the following.

\subsection{Case of a plate}

Let us consider a thin elastic plate. For this kind of structures, starting from a priori hypotheses on the expression of the displacement fields, a two-dimensional problem is usually derived from the three-dimensional elasticity formulation by means of integration along the thickness, say $2 \varepsilon$. For the Kirchhoff-Love plate model, the only variable is the normal deflection, say $u(x, t)$, and is set down on the mid-plane of the plate $\Omega$. So the Kirchhoff-Love elastodynamical model reads as

$$
\left\{\begin{array}{l}
\text { Find } u=u(x, t) \text { with }(x, t) \in \Omega \times(0, T] \text { such that for any } w \in W \\
\int_{\Omega} 2 \rho \varepsilon \frac{\partial^{2} u}{\partial t^{2}} w d x+a(u, w)=\int_{\Omega} f w d x
\end{array}\right.
$$

where

$$
a(u, w)=\int_{\Omega} \frac{2 E \varepsilon^{3}}{3\left(1-\nu^{2}\right)}\left[(1-\nu) \frac{\partial^{2} u}{\partial x_{\alpha} \partial x_{\beta}}+\nu \Delta u \delta_{\alpha \beta}\right] \frac{\partial^{2} w}{\partial x_{\alpha} \partial x_{\beta}} d x,
$$

where the mechanical constants, for a plate made of a homogeneous and isotropic material, are its Young modulus $E$, its Poisson ratio $\nu$ and its mass density $\rho$. Moreover, $\delta_{\alpha \beta}$ is the Kronecker symbol and the summation convention over repeated indices is adopted, Greek indices varying in $\{1,2\}$. If the plate is assumed to be clamped on a non-zero measure part of the boundary $\partial \Omega$ denoted $\Gamma_{c}$ and free on $\Gamma_{f}$, such as $\partial \Omega=\Gamma_{c} \cup \Gamma_{f}$, the space of admissible displacements is

$$
W=\left\{w \in H^{2}(\Omega) / w(x)=0=\partial_{n} w(x), \forall x \in \Gamma_{c}\right\}
$$


where $\partial_{n} w$ is the normal derivative along $\Gamma_{c}$. Finally, the associated initial conditions are

$$
u(x, 0)=u_{0}(x), \frac{\partial u}{\partial t}(x, 0)=v_{0}(x), \forall x \in \Omega
$$

Let us now introduce the dynamic frictionless Kirchhoff-Love equation with Signorini contact conditions along the plate. We assume that the plate motion is also limited by rigid obstacles located above and below the plate. So, the displacement is constrained to belong to the convex set

$$
K=\left\{w \in W: g_{1}(x) \leq w(x) \leq g_{2}(x), \forall x \in \Omega\right\}
$$

where $g_{1}$ and $g_{2}$ are two maps which still satisfy $g_{1}(x)<0<g_{2}(x)$, for all $x \in \Omega$. Then, the mechanical frictionless elastodynamic problem for a plate between two rigid obstacles can be written as the following variational inequality

$$
\left\{\begin{array}{l}
\text { Find } u:[0, T] \rightarrow K \text { and } v:[0, T] \rightarrow L^{2}(\Omega) \text { such for a.e. } t \in(0, T] \\
\int_{\Omega} 2 \rho \varepsilon \frac{\partial v}{\partial t}(w-u) d x+a(u, w-u) \geq \int_{\Omega} f(w-u) d x \quad, \quad \forall w \in K \\
\int_{\Omega}\left(v-\frac{\partial u}{\partial t}\right) q d x=0, \forall q \in L^{2}(\Omega), \\
u(x, 0)=u_{0}(x) \in K \quad, \quad \frac{\partial u}{\partial t}(x, 0)=v_{0}(x), \forall x \in \Omega
\end{array}\right.
$$

Let us now introduce the space discretization of the displacement. As the Kirchhoff-Love model corresponds to a fourth order partial differential equation, a conformal finite element approximation needs the use of continuously differentiable elements. Among such ones (see [2]), the reduced HCT (Hsieh-Clough-Tocher) triangles and FVS (Fraeijs de Veubeke-Sanders) quadrangles are of particular interest. For the HCT (resp. FVS) element, the triangle (resp. quadrangle) is divided into three (resp. four) sub-triangles. The basis functions of these elements are $P_{3}$ polynomials on each subtriangle and matched $\mathscr{C}^{1}$ across each internal edge. In addition, to decrease the number of degrees of freedom, the normal derivative is assumed to vary linearly along the external edges of the elements. Finally, both for triangles and quadrangles, there are only three degrees of freedom on each node: The value of the function and its first derivatives.

In [24], such elements for the deflection, piecewise constant velocity and still a nodal contact condition (as for beams) on each vertex of the mesh are numerically shown to satisfy the inf-sup condition (5).

\section{NuMERICAL DISCUSSION}

\subsection{Midpoint schemes}

As far as numerical results are concerned, in this paper, we mainly use a midpoint scheme for the time discretization of the problem. It is an interesting scheme since it is energy conserving on the linear part (equation without constraint) but, of course, any other stable scheme can be applied. For exemple, in [23] and [24], Newmark schemes are also used. So, if $\Delta t$ stands for the time step, the midpoint scheme, applied on all the previous problems, consists in finding $U^{n+1 / 2}$ in $K^{h}$ such that

$$
\left\{\begin{array}{l}
\left(W-U^{n+1 / 2}\right)^{T}\left(M Z^{n+1 / 2}+A U^{n+1 / 2}\right) \geq\left(W-U^{n+1 / 2}\right)^{T} F^{n}, \forall W \in K^{h}, \\
U^{n+1 / 2}=\frac{U^{n}+U^{n+1}}{2}, V^{n+1 / 2}=\frac{V^{n}+V^{n+1}}{2}, \\
B U^{n+1}=B U^{n}+\Delta t C V^{n+1 / 2}, C V^{n+1}=C V^{n}+\Delta t B Z^{n+1 / 2}
\end{array}\right.
$$

where $M$ and $A$ are the mass and the stiffness matrices corresponding to each discretized problem, while $Z^{n+1 / 2}$ is the acceleration at "middle time step" $n+1 / 2$ and $V^{k}$ an approximation of the 
velocity at time $k \Delta t$. As matrix $C$ is invertible, we have

$$
V^{n+1}=2 V^{n+1 / 2}-V^{n}=2 C^{-1} B \frac{U^{n+1}-U^{n}}{\Delta t}-V^{n}=4 C^{-1} B \frac{U^{n+1 / 2}-U^{n}}{\Delta t}-V^{n}
$$

Moreover, $Z^{n+1 / 2}$ can be eliminated in the following way

$$
M Z^{n+1 / 2}=B^{T} C^{-1} B Z^{n+1 / 2}=B^{T} C^{-1} \frac{C V^{n+1}-C V^{n}}{\Delta t}=B^{T} \frac{V^{n+1}-V^{n}}{\Delta t}
$$

or more explicitly

$M Z^{n+1 / 2}=4 B^{T} C^{-1} B \frac{U^{n+1 / 2}-U^{n}}{\Delta t^{2}}-2 B^{T} \frac{V^{n}}{\Delta t}=\frac{4}{\Delta t^{2}} M U^{n+1 / 2}-\frac{4}{\Delta t^{2}} M U^{n}-\frac{2}{\Delta t} B^{T} V^{n} S$.

Then, a new formulation of $(7)$ is

$$
\left\{\begin{array}{l}
U^{n} \text { and } V^{n} \text { being given, find } U^{n+1 / 2} \in K^{h} \text { such that } \\
\left(W-U^{n+1 / 2}\right)^{T}\left(\frac{4}{\Delta t^{2}} M U^{n+1 / 2}+A U^{n+1 / 2}\right) \geq\left(W-U^{n+1 / 2}\right)^{T} \bar{F}^{n}, \forall W \in K^{h} \\
\text { where } \bar{F}^{n}=F^{n}+\frac{4}{\Delta t^{2}} M U^{n}+\frac{2}{\Delta t} B^{T} V^{n} \\
U^{n+1}=2 U^{n+1 / 2}-U^{n}, V^{n+1}=2 C^{-1} B \frac{U^{n+1}-U^{n}}{\Delta t}-V^{n}
\end{array}\right.
$$

Let us note that this variational inequality has always a unique solution even if $M$ is singular.

\subsection{Case of the membrane model}

We present now some numerical experiments on the membrane problem, with

$$
\Omega=(0,1) \times(0,1), \Gamma_{D}=\partial \Omega, \Gamma_{N}=\emptyset, f=-0.6
$$

The initial condition is $u(x, 0)=0.02, \frac{\partial u}{\partial t}(x, 0)=0$, for all $x \in \Omega$, and we consider a nonhomogeneous Dirichlet condition $u(x, t)=0.02$, for all $x \in \partial \Omega$.

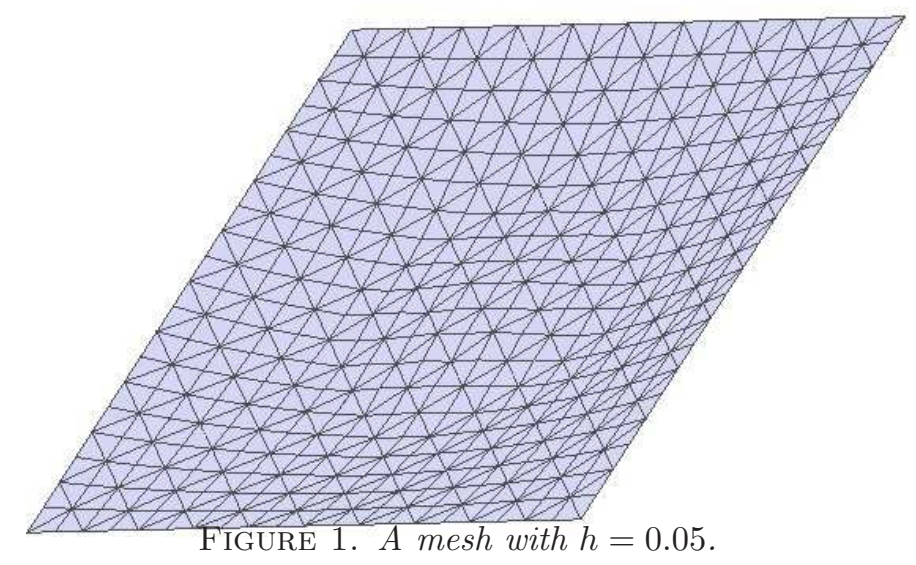




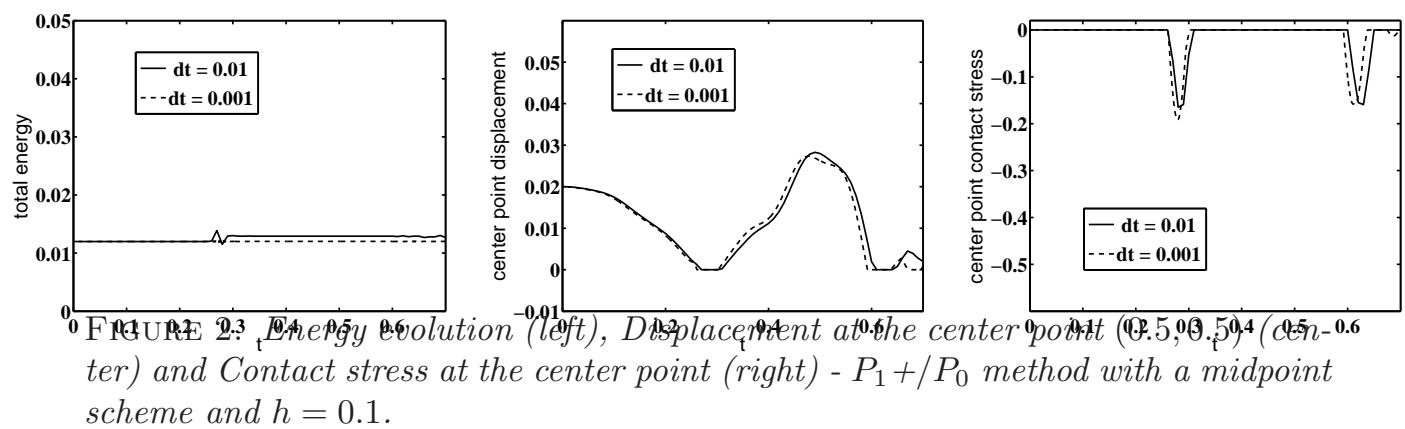

The mesh, we used, is structured and can be viewed on Figure 1, where the solution is represented during the first impact on the obstacle. The numerical experiments are performed with our finite element library Getfem $++[26]$. A semi-smooth Newton method is used to solve the discrete problem (see $[1,13])$. All the numerical experiments use the same definition of convex $K^{h}$, given by $(6)$.

The first numerical test is made with the midpoint scheme and the approximation presented in Section 3 , that is a $P_{1}+/ P_{0}$ method $\left(P_{1}+\right.$ for displacement and $P_{0}$ for velocity).

In good accordance with the theoretical results, the curves on Figure 2 show that the energy tends to be conserved when the time step decreases (an experiment with $\Delta t=10^{-4}$ has been performed but the difference with the one for $\Delta t=10^{-3}$ is not visible). Moreover, both the displacement and the contact stress, taken at the point $(0.5,0.5)$, are smooth and converge satisfactorily when the time step decreases.

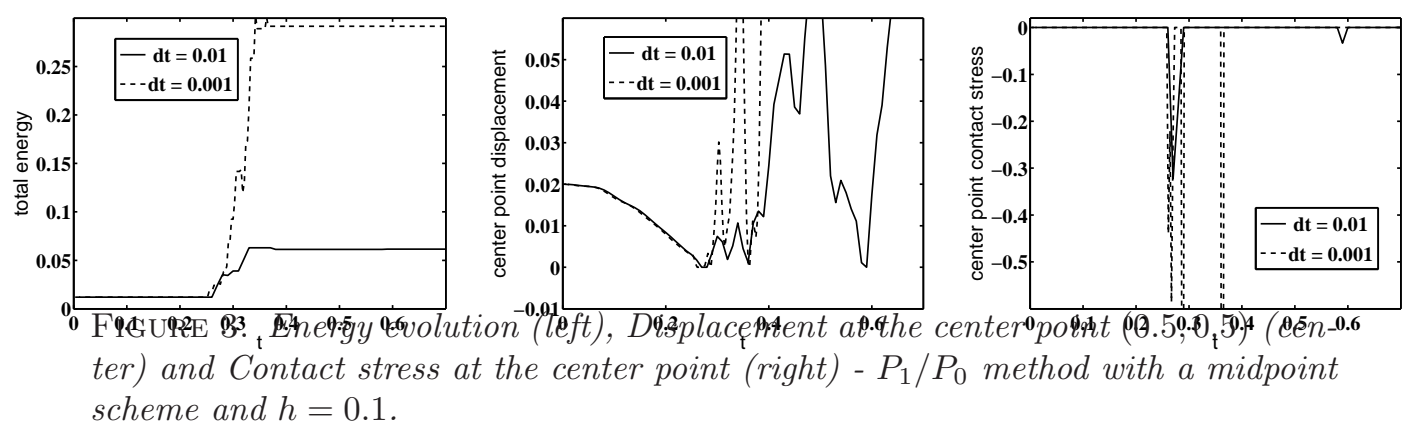

Conversely, the curves on Figure 3, obtained for a $P_{1} / P_{0}$ method, are unstable. The energy is growing very fast after the first impact. The displacement and the contact stress are very oscillating and do not converge. Moreover, the instabilities are more important for the smallest time step. This method does not satisfy the condition (5) since $\operatorname{dim}\left(H^{h}\right) \geq \operatorname{dim}\left(W^{h}\right)$.

An interesting situation is also presented in Figures 4, 5 and 6, where a backward Euler scheme is used. This time integration scheme is unconditionally stable because it is possible to prove that the discrete energy decreases from an iteration to another (see [11] for instance). This is the case for any choice of $W^{h}$ and $H^{h}$. Consequently, this method presents some smooth results for the displacement and the contact stress. However, the energy decreases rapidly for large time steps. Figure 4 shows that for a well-posed method, the energy tends to be conserved for small time steps, but Figures 5 and 6 show that, with an ill-posed method (such as classical discretizations), there is an energy loss at the impact which does not vanish when the time step and the mesh size decrease. This means that with an ill-posed method, we do not approximate a physical solution of the problem whenever one expects energy conservation to be satisfied at the limit. 


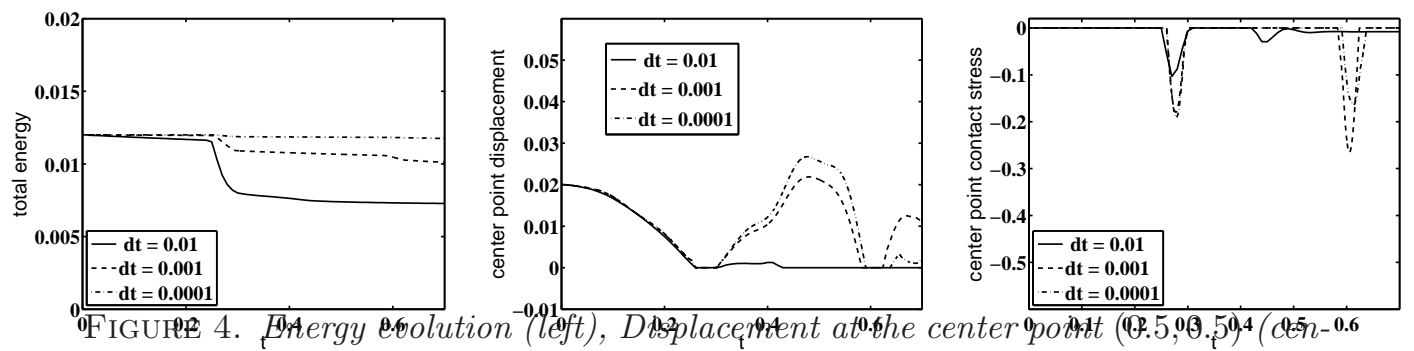

ter) and Contact stress at the center point (right) $-P_{1}+/ P_{0}$ method with a backward

Euler scheme and $h=0.1$.
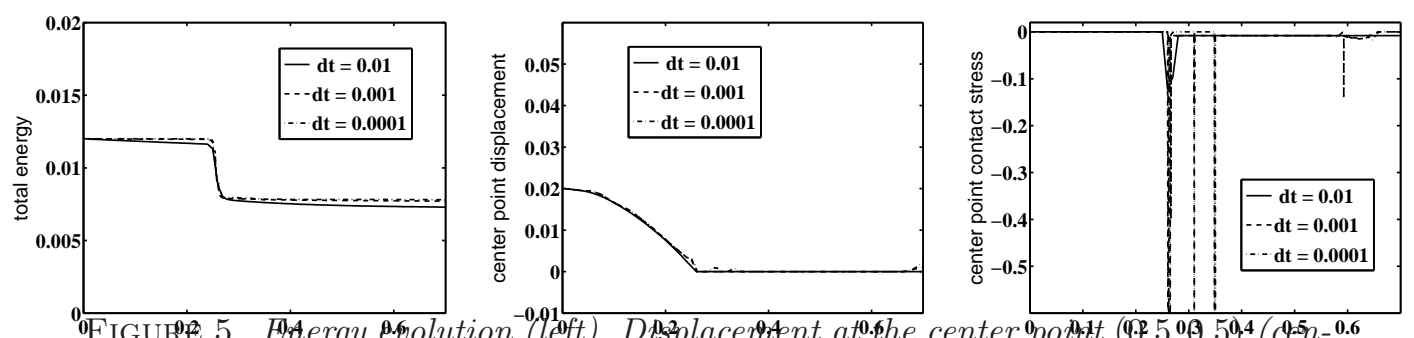

ter) and Contact stress at the center point (right) - $P_{1} / P_{0}$ method with a backward

Euler scheme and $h=0.1$.

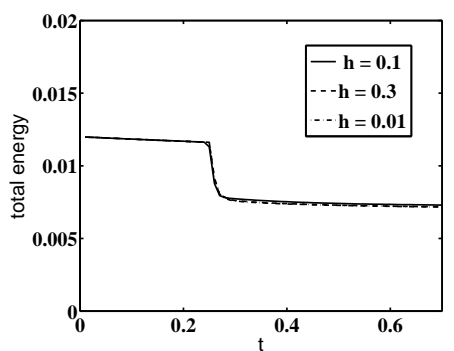

FiguRE 6. Energy evolution for a $P_{1} / P_{0}$ method, a backward Euler scheme and $\Delta t=0.001$, for different values of the mesh size.

\subsection{Case of a beam}

As in Dumont-Paoli [22], it is considered the case of a steel pipe, which length is $L=1.501 \mathrm{~m}$, external diameter is equal to $1 \mathrm{~cm}$ and thickness is $0.5 \mathrm{~mm}$. The material properties are characterized by its Young modulus $E=2.10^{11} \mathrm{~Pa}$ and its density $\rho=8.10^{3} \mathrm{~kg} / \mathrm{m}^{3}$. Thus, in this case, we have $\frac{E I}{\rho S}=282.84 \mathrm{~m}^{4} . \mathrm{s}^{-2}$, where $I$ is the quadratic momentum of inertia of the beam and $S$ its section. Moreover, in the following, we will consider flat obstacles all along the beam

$$
g_{2}(x)=-g_{1}(x)=0.1, \forall x \in[0, L]
$$

The midpoint scheme is used here, associated with the use of the cubic Hermite element for the deflection and either a piecewise constant finite element method (Figure 7), or a continuous linear one (Figure 8), for the velocity. Here again, the curves show that the energy tends to be conserved when the time step decreases in each case. 


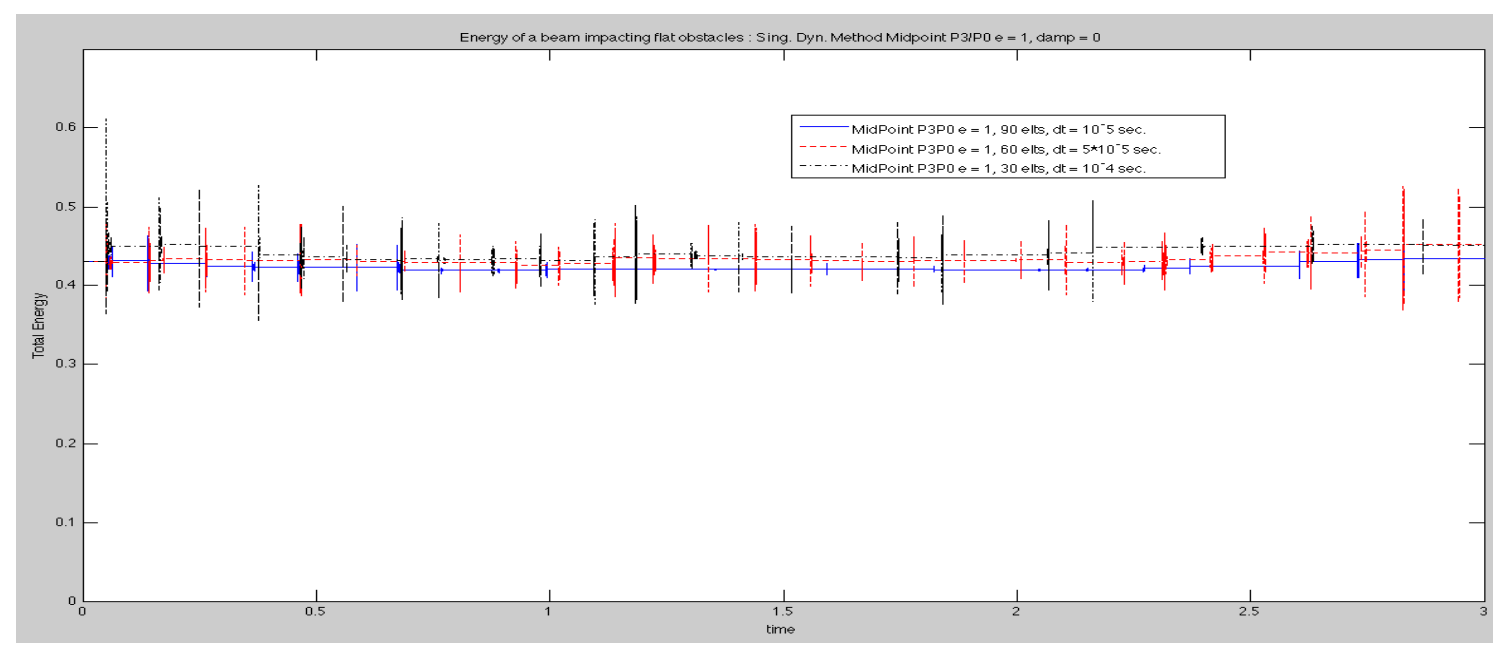

FiguRE 7. Energy for different time steps - P3/P0 singular mass matrix for Midpoint scheme. $\Delta t=10^{-4}, 5.10^{-5}, 10^{-5}, 30 / 60 / 90$ elements.

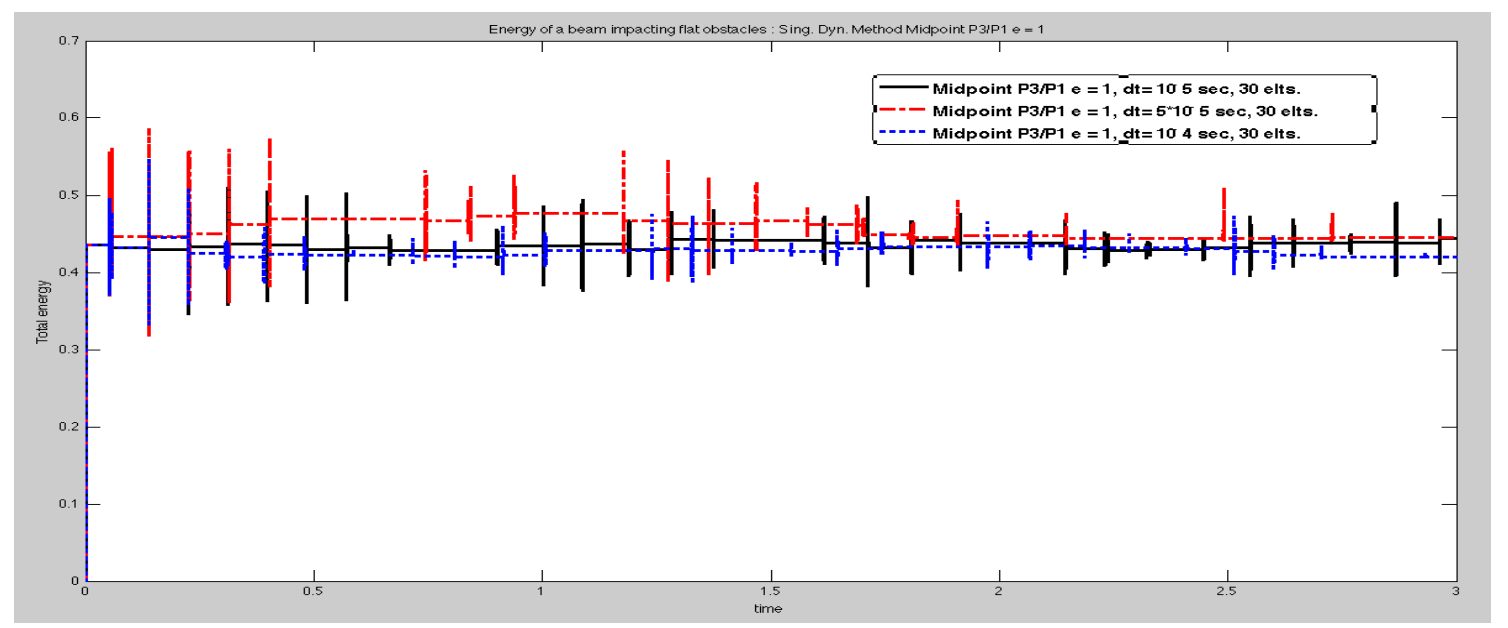

FiguRE 8. Energy for different time steps - P3/P1 singular mass matrix for Midpoint scheme. $\Delta t=10^{-4}, 5.10^{-5}, 10^{-5}$, 30 elements.

\subsection{Case of a plate}

A steel rectangular panel is considered, of length $L=120 \mathrm{~cm}$, width $l=40 \mathrm{~cm}$ and thickness $\varepsilon=0.5 \mathrm{~cm}$. It means domain $\Omega$ is $] 0, L[\times] 0, l\left[\right.$. The flexural rigidity is $D=1.92310^{4}$, corresponding to $E=210 \mathrm{GPa}$ and $\nu=0.3$, while $\rho=7.7710^{3} \mathrm{~kg} / \mathrm{m}^{3}$. This plate is clamped along one edge and free along the three others. Moreover, only the following kind of obstacle will be considered here. It is a flat obstacle under the whole plate, which reads

$$
g_{2}\left(x_{1}, x_{2}\right)=+\infty \quad, \quad g_{1}\left(x_{1}, x_{2}\right)=-0.1 \quad, \quad \forall\left(x_{1}, x_{2}\right) \in \Omega .
$$


Finally, as we are mainly interested to study conservation of energy, we consider the case where there is no loading $f(x, t) \equiv 0$ for all $x$ and $t$. All energy is contained in an initial displacement $u_{0}$, obtained as the static equilibrium of the plate under a constant load $f_{0}=14600 \mathrm{~N}$ and an initial velocity $v_{0}=0$.

In the following figures are given the energy evolutions for different time steps, for midpoint scheme with singular mass matrix. We notice that energy is weakly increasing and can be stabilized when the time step decreases. And the numericaly observed stability condition seems to be more restrictive for triangles than for quadrilaterals.

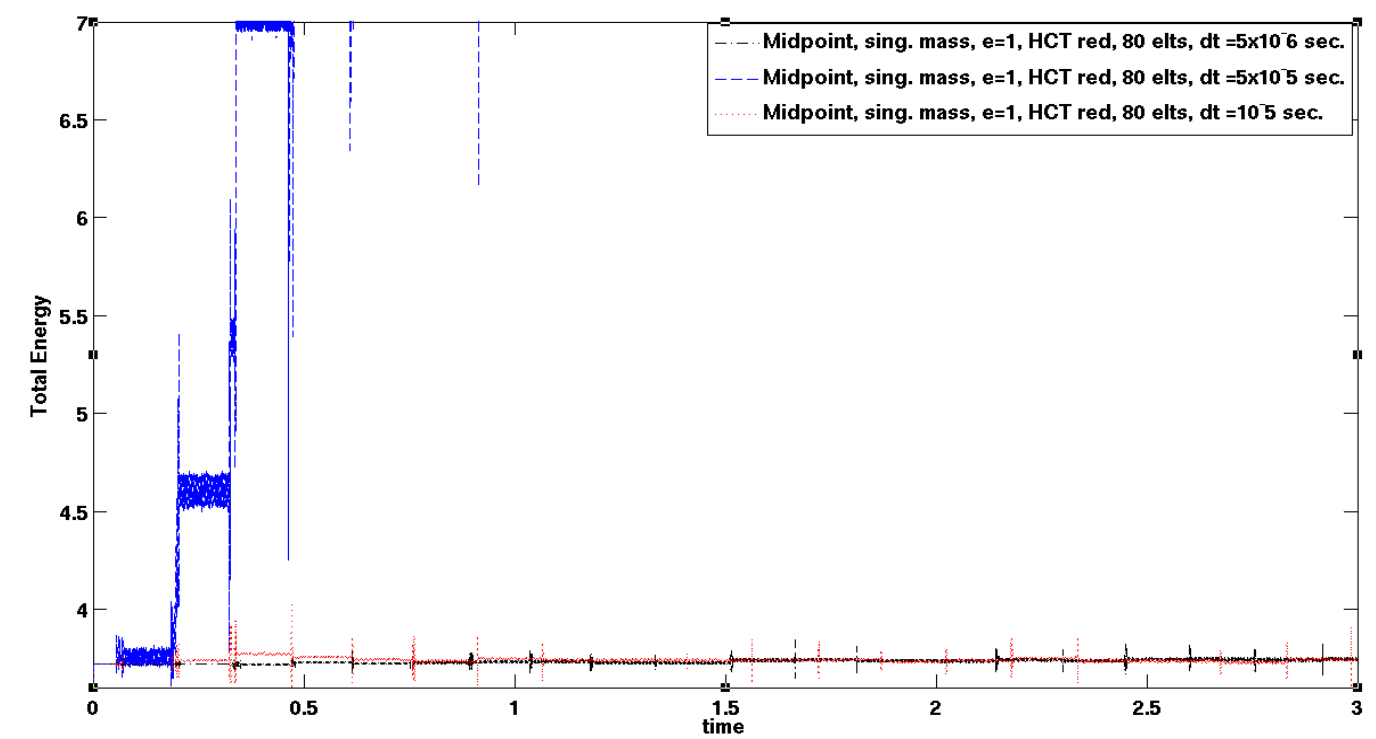

Figure 9. Energy for different time steps. Reduced HCT , 80 triangles. Midpoint scheme. 


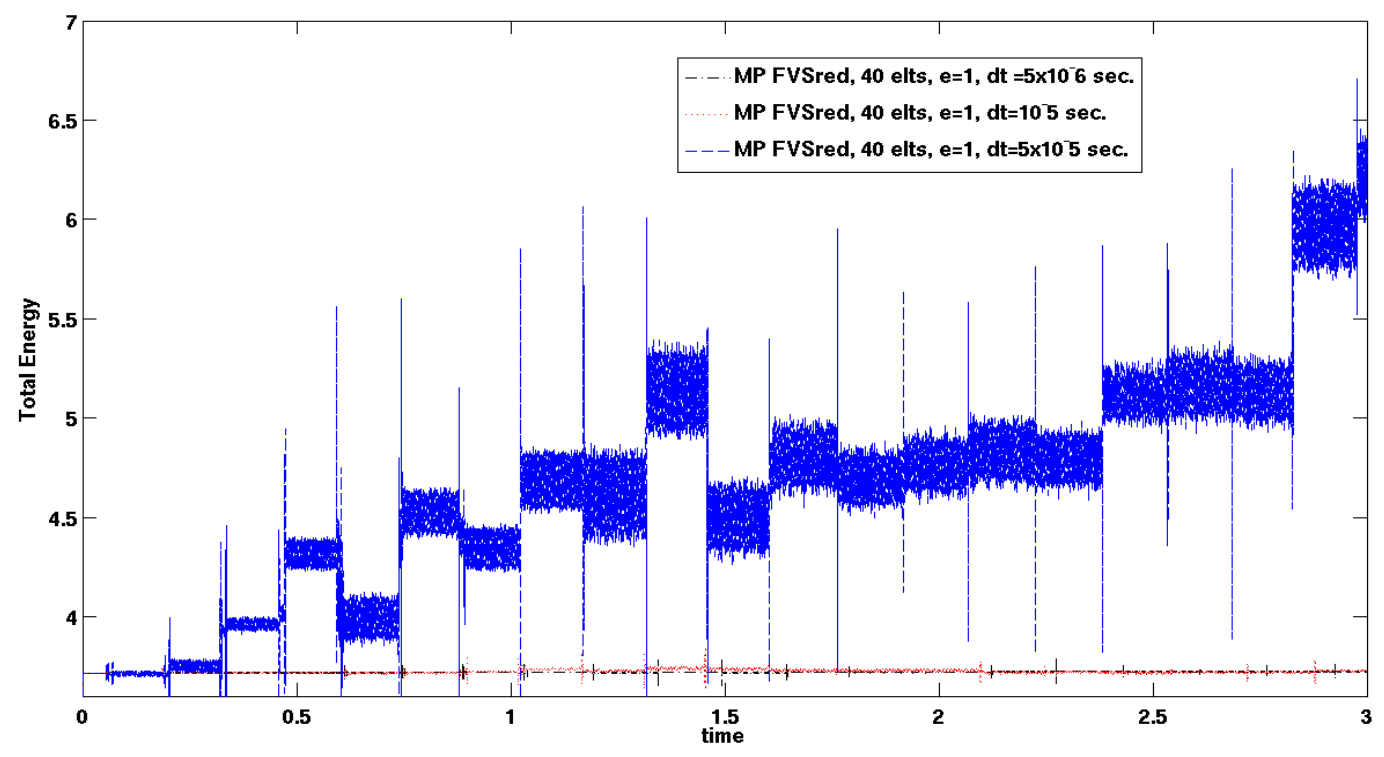

FiguRE 10. Energy for different time steps. Reduced FVS , 40 quadrilaterals. Midpoint scheme.

\section{Open PROBLEMS AND PERSPECTIVES}

The semi-discretization, proposed here, leads to a problem which is equivalent to a regular Lipschitz ordinary differential equation (see also [29] for a slightly different approach). This method generalizes in a sense the ones presented in $[6,12]$ with the advantage that no artificial modification of the mass matrix is necessary.

This is compared to the classical semi-discretizations, for example with finite element methods, which give a problem in time which is a measure differential inclusion (see [18-21]). Such a differential inclusion is systematically ill-posed, unless an additional impact law is considered.

Concerning thin structures, as it is illustrated by Figures 4, 5 and 6, numerical schemes do not necessarily converge toward the same solution. The limit solution may have different characteristics of impact energy loss. This suggests that in the case of thin structures, modeling of the restitution of the impact energy should be added to the impact law. The proposed semi-discretization being conservative in energy, it corresponds to a total restitution of the impact energy. A classical semidiscretization by finite elements with an implicit Euler scheme, as in Figure 6 corresponds to a certain loss of impact energy. Note that this energy loss is not necessarily maximal. Finally, the dissipation of the impact will certainly depend at the same time on the type of semi-discretization in space, the type of time integration scheme, the ratio between the space step and the time step, the kind of discretization of the contact conditions and finally of how the structure impacts the thin rigid obstacle (more or less obliquely, for instance). For the moment, the accurate modeling of the energy restitution at impact for the approximation of the dynamics of thin structures seems a little studied area. An interesting perspective is to try to characterize the different numerical schemes according to their characteristic in term of energy restitution.

\section{REFERENCES}

[1] P. Alart, A. Curnier. A mixed formulation for frictional contact problems prone to Newton like solution methods. Comp. Meth. Appl. Mech. Engng., 92, pp. 353-375, 1991.

[2] P.G. Ciarlet. The finite element method for elliptic problems. Studies in Mathematics and its Applications No 4, North Holland, 1978.

[3] K. Deimling. Multivalued Differential Equations. de Gruyter, 1992.

[4] P. Deuflhard, R. Krause, S. Ertel. A Contact-Stabilized Newmark Method for dynamical contact problems. Int. J. Num. Meth. Eng, 73(9), pp. 1274-1290, 2007. 
[5] N.J. Carpenter. Lagrange constraints for transient finite element surface contact. Int. J. Num. Meth. Eng, 32, pp. 103-128, 1991.

[6] C. Hager, B. Wohlmuth. Analysis of a modified mass lumping method for the stabilization of frictional contact problems. to appear in SIAM J. Numer. Anal.

[7] P. HAuret. Numerical methods for the dynamic analysis of two-scale incompressible nonlinear structures. Thèse de Doctorat, Ecole Polythechnique, France, 2004.

[8] P. Hauret, P. Le TAllec. Energy controlling time integration methods for nonlinear elastodynamics and lowvelocity impact. Comput. Meth. Appl. Mech. Engrg., 195, pp. 4890-4916, 2006.

[9] T.J.R. Hugues, H. M. Hiler And R. L. TAYlor. A reduction scheme for problems of structural dynamics. 12 , pp. 749-767, 1976.

[10] T.J.R. Hugues, R. L. Taylor, J. L. Sackman, A. Curnier, W. Kanok-Nukulchai. A finite element method for a class of contact-impact problems. 8, pp. 249-276, 1976.

[11] H.B. Khenous. Problèmes de contact unilatéral avec frottement de Coulomb en élastostatique et élastodynamique. Etude mathématique et résolution numérique. PhD thesis, INSA de Toulouse, France, 2005.

[12] H.B. Khenous, P. LABorde, Y. RenARd. Mass redistribution method for finite element contact problems in elastodynamics. Eur. J. Mech., A/Solids, 27(5), pp. 918-932, 2008.

[13] H.B. Khenous, J. Pommier, Y. Renard. Hybrid discretization of the Signorini problem with Coulomb friction. Theoretical aspects and comparison of some numerical solvers. Applied Numerical Mathematics, 56(2), pp. 163$192,2006$.

[14] J.U. KIm. A boundary thin obstacle problem for a wave equation. Com. part. diff. eqs., 14(8\&9), pp. 1011-1026, 1989.

[15] T.A. Laursen, V. Chawla. Design of energy conserving algorithms for frictionless dynamic contact problems. Int. J. Num. Meth. Engrg, 40, pp. 863-886, 1997.

[16] T.A. LAursen, G.R. Love. Improved implicit integrators for transient impact problems-geometric admissibility within the conserving framework. Int. J. Num. Meth. Eng., 53, pp. 245-274, 2002.

[17] G. Lebeau, M. Schatzman. A wave problem in a half-space with a unilateral constraint at the boundary. J. diff. eqs., 55, pp. 309-361, 1984.

[18] J.J. Moreau. Liaisons unilatérales sans frottement et chocs inélastiques. C.R.A.S. série II, 296, pp. 1473-1476, 1983.

[19] J.J. Moreau. Numerical aspects of the sweeping process. Comp. Meth. Appl. Mech. Engrg., 177, pp. 329-349, 1999.

[20] L. Paoli. Time discretization of vibro-impact. Phil. Trans. R. Soc. Lond. A., 359, pp. $2405-2428,2001$.

[21] L. Paoli, M. Schatzman. Approximation et existence en vibro-impact. C. R. Acad. Sci. Paris, Sér. I, 329, pp. 1103-1107, 1999.

[22] Y. Dumont, L. Paoli. Vibrations of a beam between obstacles: convergence of a fully discretized approximation. M2AN, 40(4), pp. 705-734, 2006.

[23] C. Pozzolini, M. Salaun. Some Energy conservative schemes for vibro-impacts of a beam on rigid obstacles. ESAIM: M2AN, 45, pp. 1163-1192, 2011.

[24] C. Pozzolini, Y. Renard, M. Salaun. Asymptotic energy preserving schemes schemes for the vibro-impacts of a plates between rigid obstacles. Submitted.

[25] Y. RENARD. The singular dynamic method for constrained second order hyperbolic equations. Application to dynamic contact problems. J. Comput. Appl. Math., 234(3), pp. 906-923, 2010.

[26] Y. Renard, J. Pommier. Getfem ++ . An Open Source generic C++ library for finite element methods. http://home.gna.org/getfem.

[27] K. Schweizerhof, J.O. Hallquist, D. Stillman. Efficiency Refinements of Contact Strategies and Algorithms in Explicit Finite Element Programming. In Computational Plasticity, eds. Owen, Onate, Hinton, Pineridge Press, pp. 457-482, 1992.

[28] R. L. TAylor, P. Papadopoulos. On a finite element method for dynamic contact-impact problems. Int. J. for Num. Meth. Eng., 36, pp. 2123-2140, 1993.

[29] A.Tkachuk, B. I. Wohlmuth, M. Bischoff. Hybrid-mixed discretization of elasto-dynamic contact problems using consistent singular mass matrices. to appear in Int. J. for Num. Meth. Eng., DOI: 10.1002/nme, 2013. 